\title{
PRODUCTION OF DIFFERENT NON-THERMAL ELECTRON GROUPS IN SMALL SOLAR FLARES*
}

\author{
S. R. KANE \\ Space Sciences Laboratory, University of California, \\ Berkeley, Calif. 94720, U.S.A.
}

Using the measurements of impulsive solar X-rays made with the OGO-5 satellite to identify the flash phase electron acceleration in solar flares of $\mathrm{H} \alpha$-importance $\lesssim 1$, the satellite and ground based observations are analyzed to study the origin of the different groups of non-thermal electrons responsible for the impulsive X-ray, impulsive microwave, type III radio and interplanetary electron emission. The observational results may be summarized as follows:

(1) About $68 \%$ of the impulsive X-ray bursts have associated impulsive microwave bursts. In the remaining $32 \%$ cases, it is possible that the microwave emission is too small to be detected above the existing background level. In most of the correlated events the microwave maximum occurs within $\pm 6 \mathrm{~s}$ of the $\mathrm{X}$-ray maximum. The observations are consistent with an essentially simultaneous occurrence of the X-ray and microwave maxima.

(2) About $37 \%$ of the impulsive X-ray bursts have associated type III radio bursts. In a large number of the correlated events, the onset of the type III burst occurs within \pm 1 min of the onset of the X-ray burst.

(3) In a solar flare the probability of occurrence of non-thermal electron density spectrum with a particular power law exponent $\varphi \$ 4$ is found to be nearly the same whether the probability is deduced from the observations of the impulsive X-ray bursts or the interplanetary electron events. For $\varphi>4$ the probability of occurrence deduced from the two sets of observations is different. But this difference can be explained in terms of the hardening of the electron spectrum during its escape from the flare region and/or during its propagation to a distance of $1 \mathrm{AU}$.

The above observations are consistent with the following interpretation. During the flash phase of a solar flare, a population of non-thermal electrons is produced in a region such as the base of the corona where the ion density is small and consequently the bremsstrahlung X-ray emission is also small. After a trapping time $\lesssim 1$ minute the electrons tend to escape from this region and propagate in the solar atmosphere guided by the magnetic field structure. The trapping time may depend on the direction of eventual escape from the production region. The group of electrons moving towards the denser parts of the flare give rise to the impulsive X-ray and microwave emission. The group of electrons moving towards lower density regions produce type III radio bursts. Some electrons escape completely from the solar atmosphere and are observed in interplanetary space by suitably located detectors. The above interpretation is con-

* Research supported by NASA under Contract NAS 5-9094. 
sistent with models proposed, for example, by de Jager and Kundu(1963), Wild (1964) and Anderson et al. (1970).

\section{Acknowledgement}

Research supported by the National Aeronautics and Space Administration under contract NAS 5-9094.

\section{References}

Anderson, K. A., Kane, S. R., and Lin, R. P.: 1970, Fast Electrons from Solar Flares, Presented at the International Seminar on the Problem of Cosmic Ray Generation on the Sun, Leningrad, U.S.S.R., December 1970 (UC Space Sciences Lab, Series 12, Issue 7).

De Jager, C. and Kundu, M. R.: 1963, in W. Priester (ed.), Space Res. 3, 836, North-Holland Publ. Co., Amsterdam.

Wild, J. P.: 1964, AAS-NASA Symposium on Solar Flares, NASA SP-50.

\section{DISCUSSION}

$Z$. Svestka: There are many type III bursts. Only a few of them are associated with microwave bursts. I would like to know whether the type III bursts entering your statistics were all associated with microwave bursts or not (i.e. coinciding in time with them so that one burst extended along the whole spectrum).

S. R. Kane: Most of the type III bursts were associated with microwave bursts but the two were not necessarily coincident in time. The microwave bursts were coincident with the X-ray bursts. In most cases the type III bursts did not extend to centimeter wavelengths.

H. J. Kunze: You discussed clear evidence for non thermal celectrons in solar flares. These electrons, however, should excite plasma waves at the exectron plasma frequency $\omega_{p}$ which in principle are observable using '2nd order spectroscopy' as defined by Burgess (this issue, p. 493.) More specifically this means, one should observe 'plasma satellite lines' displaced by $2 \omega_{\mathrm{p}}$ from allowed lines (perhaps $\mathbf{H} \alpha, \mathbf{H} \beta$ ) or others displayed by $\omega_{\mathrm{p}}$ from forbidden lines $(\Delta l=0,2)$, for example HeI lines. My question to you, or perhaps to the general audience is: Have such plasma satellites ever been observed in solar flares, or why not? Besides obtaining the level of excitation of such waves one would also obtain a very accurate determination of the electron density.

S. R. Kane: The observations that I discussed here do not refer to line emission. Somebody who makes such measurements should perhaps answer your question.

C. de Jager: The type III bursts are probably due to jets of electrons moving upward through the corona with velocities, smaller but comparable to the speed of light. Their occurrence must be related to plasma instabilities in the flowing region and to the magnetic field configuration, which should be 'open' in such a way as to permit the upward motion of the electron jets. On the other hand the microwave and $\mathrm{X}$ radiation, which as your statistics show are as closely correlated in the times of their occurrence, may be due to accelerated particles trapped in the lower coronal regions.

Your observation that the correlation of type III bursts with X bursts is much looser, with type III's occurring already some 3-4 min. before the flares onset, shows in a dramatic way the capability of the flare region to produce already occasional upward moving jets of electrons, at a time when the large acceleration process, that gives rise to the flare proper, is not yet occurring.

S. R. Kane: An alternative explanation can be as follows. The flare process has occurred in that the electrons have been accelerated as implied by the type III burst. The reason for the X-ray burst being observed several minutes after the type III burst could be that some of the accelerated electrons were trapped in the acceleration region for several minutes before they could escape and penetrate into the denser regions of the flare, producing X-rays. 